\title{
Deutsche Röntgengesellschaft verleiht „Young Investigator Award“
}

Die Deutsche Röntgengesellschaft hat in diesem Jahr 2 Nachwuchswissenschaftler mit dem „Young Investigator Award“ ausgezeichnet. Die Preisträger sind PD Dr. Sven Nebelung aus Aachen/ Düsseldorf und Eckhard Wehrse aus Heidelberg. Mit dem „Young Investigator Award" hebt die Fachgesellschaft alljährlich die Leistungen des wissenschaftlichen Nachwuchses in der Bildgebung heraus und fördert ihn.

Die Deutsche Röntgengesellschaft hat ihren Preis „Young Investigator Award“ für junge Nachwuchswissenschaftlerinnen und -wissenschaftler in diesem Jahr an PD Dr. Sven Nebelung und Eckhard Wehrse verliehen. Dr. Sven Nebelung wurde für seine Arbeit mit dem Titel „Automatisierte Bemaßung des biomechanischen Alignments von Ganzbeinstandröntgenuntersuchungen “ ausgezeichnet. Das mit dem Preis ausgezeichnete Projekt hat Dr. Nebelung an den beiden Universitätsklinika Aachen (Klinik für Diagnostische und Interventionelle Radiologie) und Düsseldorf (Institut für Diagnostische und Interventionelle Radiologie) umgesetzt. Der Titel der ausgezeichneten Arbeit von Eckhard Wehrse ist „Detektion von Milchglasinfiltraten in der Photonenzählenden Niedrigdosis-CT“. Eckhard Wehrse ist Promovend am Deutschen Krebsforschungszentrum (Forschungsschwerpunkt Bildgebung und Radioonkologie) in Heidelberg.

Verliehen wurde der „Young Investigator Award“ im Rahmen des 102. Deutschen Röntgenkongresses. Für den Preis konnten sich Wissenschaftlerinnen und Wissenschaftler aller Fachbereiche bewerben. Aus allen Wettbewerbsbeiträgen wurden über einen Review-Prozess 6 Autorinnen und Autoren ausgewählt, die ihre Forschungsarbeit beim Deutschen Röntgenkongress vorstellen konnten.

Seit dem Jahr 2009 ehrt die Deutsche Röntgengesellschaft alljährlich anlässlich des Deutschen Röntgenkongresses herausragende Arbeiten und Kongressbeiträge junger Wissenschaftlerinnen und Wissenschaftler mit dem „Young Investigator Award“. Mit dem Preis will die Fachgesellschaft die Leistungen des wissenschaftlichen Nachwuchses in der Bildgebung herausheben und fördern. 